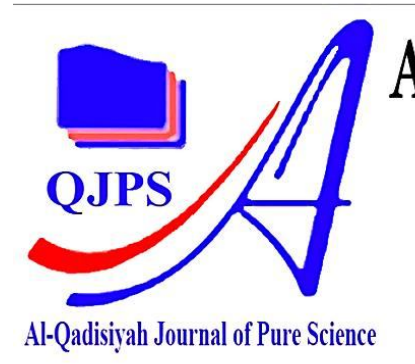

Al-Qadisiyah Journal of Pure Science

ISSN(Printed): 1997-2490

ISSN(Online):

$2411-3514$

DOI : /10.29350/jops.

http://qu.edu.iq/journalsc/index.php/JOPS

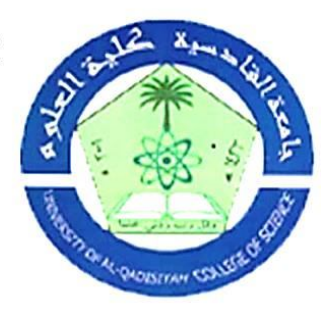

\title{
Non-autonomous invariant sets and attractors: Random dynamical system
}

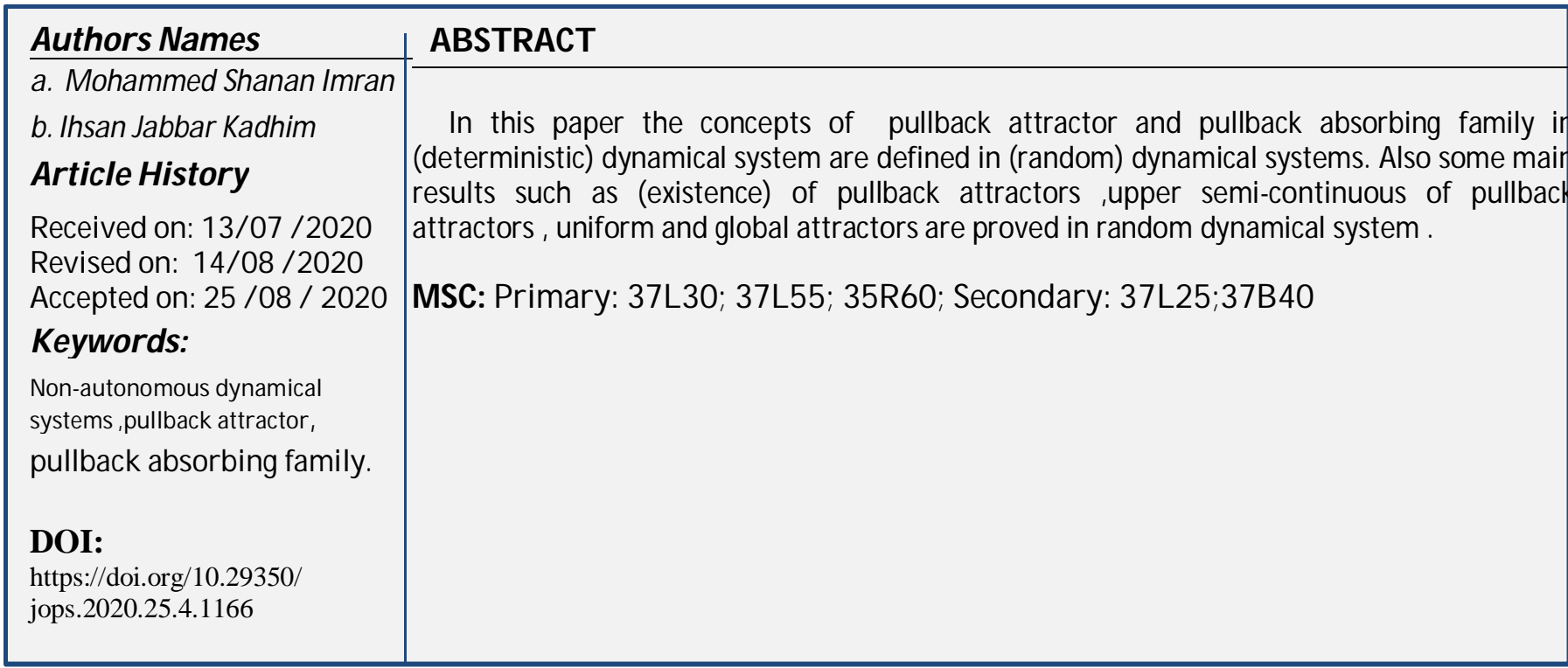

\section{Introduction}

The Nonautonomous dynamical system can frequently be expressed in expressions of a co-cycle mapping for the dynamics in the state space. That is ambitious by an autonomous dynamical system which is called a parameter space. Usually, the motivating system is topological and the subsequent Cartesian product system forms an autonomous semi-dynamical system that is recognized as a skew-product flow. Consequences, The global attractors for autonomous semi-dynamical systems can so be modified to such nonautonomous dynamical systems via the accompanying skew-product flow [1, 2, 3, 4, 7, 8, and 10]. Contemporary enlargements in random dynamical systems expands augmentation to nonautonomous dynamical systems. The motivating autonomous system is measure theoretic sooner than topological. A new kind of attractor, called a pullback attractor. It projected and scrutinized for nonautonomous or these random dynamical systems [5, 6, and 11]. Pullback attraction describes these attractors to a cog subset for a fixed parameter value which is attained by starting increasingly previously in time, that is, at parameter values that are approved forward to the fixed value. The kernels of a global attractor of the skew-product flows considered in [4] are very similar. These varies from the more conservative forward convergence where the parameter value of the restraining object to regresses with time, in which case the Parameterized family could be called a forward attractor. The association between the global attractor of the skew-product system and the pullback and forward attractors of the co-cycle system is considered in this paper. We also communicate that forward attractors are stronger than global

a Department of Mathematics, College of Science, University, Al-Qadisiyah, E-Mail: Ma.post06@qu.edu.iq 
attractors if we suppose a compact set of nonautonomous perturbations. An example is obtainable in which the Cartesian product of the cog subsets of a pullback attractor is not a global attractor of the skew-product flow. This set however, is a maximal compact invariant subset of the skew-product flow. By a generalization of some stability consequences of Zubov [11] it is asymptotically stable. Thus a pullback attractor continuously produces a local attractor of the skew-product system, but this does not need to be a global attractor. If the pullback attractor produces a global attractor in the skew-product flow and its cog subsets depend lower continuously on the parameter, then the pullback attractor is also a forward attractor. Numerous theorems and theories explain these outcomes were obtainable.

\section{Existence of pullback attractor}

Consider a discrete-time random dynamical system $(\theta, \varphi)$ on $\Omega \times \mathbb{R}^{d}$, where $(\Omega, d)$ and $\left(\mathbb{R}^{d}, d\right)$ are metric spaces. There are counterparts for random dynamical systems of the concepts of invariance, forwards and pullback convergence and forwards and pullback attractors. And consider The 5-tuple $\left(\mathbb{Z}^{+}, \Omega, \mathcal{F}, \mathbb{P}, \theta\right)$ is called a metric dynamical system (Shortly MDS), if $(\Omega, \mathcal{F}, \mathbb{P})$ is a probability space and

(i) $\quad \theta: \mathbb{Z}^{+} \times \Omega \rightarrow \Omega$ is $\left(\mathcal{B}\left(\mathbb{Z}^{+}\right) \otimes \mathcal{F}, \mathcal{F}\right)$-measurable,

(ii) $\theta(0, \omega)=I d_{\Omega}$,

(iii) $\quad \theta(m+n, \omega)=\theta(m, \theta(n, \omega))$ and

(iv) $\quad \mathbb{P}(\theta(m) F)=\mathbb{P}(F)$, for every $F \in \mathcal{F}$ and every $m \in \mathbb{Z}^{+}$.

Note that we write $\theta: \mathbb{Z}^{+} \times \Omega \rightarrow \Omega$ either in the form $\theta(m, \omega)$ ( as a function of two variable or in the form $\theta(m) \omega$. A measurable random dynamical system on the measurable space $\left(\mathbb{R}^{d}, \mathcal{B}\left(\mathbb{R}^{d}\right)\right.$ ) over (or covering, or extending) an $\operatorname{MDS}\left(\mathbb{Z}^{+}, \Omega, \mathcal{F}, \mathbb{P}, \theta\right)$ with measurability time is a mapping $\varphi: \mathbb{Z}^{+} \times \Omega \times \mathbb{R}^{d} \rightarrow$ $\mathbb{R}^{d}$, with the following properties:

(i) $\quad \varphi$ is $\mathcal{B}\left(\mathbb{Z}^{+}\right) \otimes \mathcal{F} \otimes \mathcal{B}, \mathcal{B}$ - measurable.

(ii) Cocycle property: The mappings $\varphi(m, \omega):=\varphi(m, \omega, \cdot): \mathbb{R}^{d} \rightarrow \mathbb{R}^{d}$ form a cocycle over $\theta(\cdot)$, i. e. they satisfy

$\varphi(0, \omega)=i d_{X}$ for all $\omega \in \Omega$.

$\varphi(m+n, \omega)=\varphi(m, \theta(n) \omega) \circ \varphi(n, \omega)$ for all $m, n \in \mathbb{Z}^{+}, \omega \in \Omega$.

If there is no ambiguity the $\mathrm{RDS}$ is denoted by $(\theta, \varphi)$ rather than $\left(\mathbb{Z}^{+}, \Omega, \mathbb{R}^{d}, \theta, \varphi\right)$.[9]

Definition 2.1 A collection $\mathcal{A}=\{A(\omega): \omega \in \Omega, A: \omega \mapsto A(\omega)$ be multifunction $\}$ of non-empty random sets in $\mathbb{R}^{d}$ is called $\boldsymbol{\varphi}$ invariant for random dynamical system $(\operatorname{RDS}) \quad(\theta, \varphi)$ if $\varphi(n, \omega, A(\omega))=A\left(\theta_{n} \omega\right)$ for each $n \in \mathbb{Z}^{+}, \omega \in \Omega$.

Definition 2.2 A collection $\mathcal{A}$ of non-empty compact random sets in $\mathbb{R}^{d}$ is called a pullback attractor of an $\operatorname{RDS}(\theta, \varphi)$ on $\Omega \times \mathbb{R}^{d} \quad$ if it is $\varphi$ invariant and pullback attractors bounded sets, that is $d\left(\varphi\left(j, \theta_{-j} \omega, D\left(\theta_{-j} \omega\right), A(\omega)\right) \rightarrow 0\right.$ for $j \rightarrow \infty$. 
For each $\omega \in \Omega$ and for all bounded random subsets $D(\theta \omega)$ of $\mathbb{R}^{d}$. It is called a forwards attractor if it is $\varphi$ invariant and forward attractors bounded random sets, that is,

$d\left(\varphi(j, \omega, D(\omega)), A\left(\theta_{j} \omega\right)\right) \rightarrow 0$ for $j \rightarrow \infty$.

As with processes, the existence of a pullback attractor for random dynamical system is warranted by that of pullback absorbing system.

Definition 2.3. A collection $\mathcal{B}=\{B(\omega): \omega \in \Omega\}$ random sets of nonempty compact random sets in $\mathbb{R}^{d}$ is said to be a pullback absorbing collection for $\operatorname{RDS}(\theta, \varphi)$ on $\Omega \times \mathbb{R}^{d}$ if for all $\omega \in \Omega$ and each bounded random subsets $D(\theta \omega))$ of $\mathbb{R}^{d}$ there exists an $N_{\omega, D} \in \mathbb{Z}^{+}$such that $\varphi\left(j, \theta_{-j} \omega, D\left(\theta_{-j} \omega\right)\right) \subseteq B(\theta \omega)$ for each $j \in N_{\omega, D}, \omega \in \Omega$.

Theorem 2.4. Let $\Omega$ complete and suppose that a $\operatorname{RDS}(\theta, \varphi)$ has a pullback absorbing random set collection $\mathcal{B}$. Then there exists a pullback attractor $\mathcal{A}$ with component random sets determined by

$$
A(\theta \omega)=\cap_{n \geq 0} \overline{\bigcup_{\jmath \geq n} \varphi\left(\jmath, \theta_{-\jmath} \omega, B\left(\theta_{-\jmath} \omega\right)\right)} .
$$

If the component random sets are uniformly bounded, then it is unique. The pullback attractor of an RDS $(\theta, \varphi)$ has some fine properties, if the component random subsets are contained in a common compact random subset or the state $\Omega$ of the energetic system is compact.

Theorem 2.5 .Let $\Omega$ be a metric space with a probability measure and suppose that $A(\Omega)=\overline{\cup_{\omega \in \Omega} A(\omega)}$ is compact for a pullback attractor $\mathcal{A}$. Then the set-valued mapping $\omega \mapsto A(\omega)$ is upper semi-continuous in the reason that

$d(A(\mu), A(\omega)) \rightarrow 0$,as $\mu \rightarrow \omega$, for $\mu, \omega \in \Omega$.

On the other hand, $A(\Omega)$ is compact whenever $\Omega$ is compact and the set-valued mapping $\omega \mapsto A(\omega)$ is upper semi-continuous.

Proof: By the compactness of $A(\Omega)$, the pullback attractor is uniformly bounded by a compact random sets and hence is uniquely determined.

Suppose, if possible, that the set-valued mapping $\omega \mapsto A(\omega)$ is not upper semi-continuous. There exists an $\varepsilon_{0}>0$ and a sequence $\omega_{n} \rightarrow \omega_{0}$ in $\Omega$ such that $d\left(A\left(\omega_{n}\right), A\left(\omega_{0}\right)\right) \geq 3 \varepsilon_{0}$ for every $n \in \mathbb{N}$. Since the random sets $A\left(\omega_{n}\right)$ are compact, there exists an $a_{n} \in A\left(\omega_{n}\right)$ such that 
$d\left(a_{n}, A\left(\omega_{0}\right)\right)=d\left(A\left(\omega_{n}\right), A\left(\omega_{0}\right)\right) \geq 3 \varepsilon_{0}$ for all $n \in \mathbb{N}$.

By pullback attraction, $d\left(\varphi\left(m, \theta_{-m}\left(\omega_{0}\right), B(\omega)\right), A\left(\omega_{0}\right)\right) \leq \varepsilon_{0}$ for $m \geq M_{B, \varepsilon_{0}}$ for any bounded random subset $B$ of $\mathbb{R}^{d}$, in particular, below $A(\Omega)$ will be used for random set $B$. By the $\varphi$ invariance of the pullback attractor, there exists $b_{n} \in A\left(\theta_{-m} \omega_{n}\right) \subset A(\Omega)$ for $n \in \mathbb{N}$. Such that $\varphi\left(m, \theta_{-m} \omega_{n}, b_{n}\right)=a_{n}$. Since $A(\Omega)$ is compact, there is a convergent subsequence $b_{\dot{n}^{\prime}} \rightarrow \bar{b} \in A(\Omega)$. Finally, by the continuity of $\theta_{-m}($.$) and of the cocycle mapping$ $\varphi(n, . .),. d\left(\varphi\left(m, \theta_{-m} \omega_{\dot{n}}, b_{\dot{n}}\right), \varphi\left(m, \theta_{-m} \omega_{0}, \bar{b}\right)\right) \leq \varepsilon_{0}$ for $\dot{n}$ large enough. Thus

$d\left(a_{\dot{n}}, A\left(\theta \omega_{0}\right)\right)=d\left(\varphi\left(m, \theta_{-m} \omega_{\dot{n}}, b_{\dot{n}}\right), A\left(\theta \omega_{0}\right)\right)$

$\left.\leq d\left(\varphi\left(m, \theta_{-m} \omega_{\dot{n}}, b_{\dot{n}}\right), \varphi\left(m, \theta_{-m} \omega_{0}, \bar{b}\right)\right)+d\left(\varphi\left(m, \theta_{-m} \omega_{0}\right), \bar{b}\right), A\left(\theta \omega_{0}\right)\right)$

$\leq 2 \varepsilon_{0}$.

This is a contradiction with (4). Hence $\omega \mapsto A(\omega)$ is an upper semi-continuous. The residual assertion follows from then fact that the upper semi-continuous image of a compact random subset is compact.

In addition to the suppositions of Theorem 2.5.

Theorem 2.6 let $\Omega$ is compact and let the pullback absorbing collection $\mathcal{B}$ is uniformly bounded by a compact random subset $\mathcal{C}$ of $\mathbb{R}^{d}$. Then $\lim _{n \rightarrow \infty} \sup _{\omega \in \Omega} d(\varphi(n, \omega, D(\omega)), A(\Omega))=0$

For all bounded random subset $D$ of $\mathbb{R}^{d}$, where $A(\Omega):=\overline{\mathrm{U}_{\omega \in \Omega} A(\omega)}$.

Proof: Since all the component random subsets $A(\omega)$ are contained in the common compact random set $\mathcal{C}$, then $A(\Omega)$ is compact. This means also that the pullback attractor is unique.

Suppose, if possible, that the convergence (5) does not hold. Then there exists an $\varepsilon_{0}>0$,and sequences $n_{j} \rightarrow \infty, \widehat{\omega_{j}} \in \Omega, x_{j} \in \mathcal{C}$ such that $d\left(\varphi\left(n_{j}, \widehat{\omega_{j}}, x_{j}\right), A(\Omega)\right)>\varepsilon_{0}$.

Set $\omega_{j}=\theta_{n_{j}}\left(\widehat{\omega_{j}}\right)$. By the compactness of $\Omega$, there exists a convergence subsequence $\omega_{j} \rightarrow \omega_{0} \in \Omega$. From the pullback attraction, there exists an $n>o$ such that

$d\left(\varphi\left(n, \theta_{-n}\left(\omega_{0}\right), \mathcal{C}(\omega)\right), A\left(\theta \omega_{0}\right)\right)<\varepsilon^{\varepsilon_{0}} / 2$

Then the cocycle property gives

$\left.\varphi\left(n_{j}, \theta_{-n_{j}}\left(\omega_{j}\right), x_{j}\right)\right)=\varphi\left(n, \theta_{-n}\left(\omega_{j}\right), \varphi\left(n_{j}-n, \theta_{-n_{j}}\left(\omega_{j}\right), x_{j}\right)\right)$ for any $n_{j}>n$. By the pullback absorption of $\mathcal{B}$, it follows that

$\left.\varphi\left(n_{j}-n, \theta_{-n_{j}}\left(\omega_{j}\right), x_{j}\right)\right) \subset B\left(\theta_{-n_{j}}\left(\omega_{j}\right)\right) \subset \mathcal{C}$, 
and since $\mathcal{C}$ is compact, there is a further index subsequence $\ddot{j}$ of $j$ (depending on $n$ ) such that

$z_{n_{j}}:=\varphi\left(n_{j}-n, \theta_{-n_{j}}\left(\omega_{j j}\right), x_{j}\right) \rightarrow Z_{0} \in \mathcal{C}$

Since the random mappings is continuous in the $\omega$ and $x$ variables then

$d\left(\varphi\left(n, \theta_{-n}\left(\omega_{j j}\right), Z_{n_{j}}\right), \varphi\left(n, \theta_{-n}\left(\omega_{0}\right), Z_{0}\right)\right)<\varepsilon_{0} / 2$, when $n_{j}>n\left(\varepsilon_{0}\right)$.

Therefore,

$\varepsilon_{0}>d\left(\varphi\left(n_{j}, \theta_{-n_{j}}\left(\omega_{0}\right), x_{i j}\right), A\left(\theta \omega_{0}\right)\right)$

$=d\left(\varphi\left(n_{j}, \widehat{\omega}_{j}, x_{j}\right), A\left(\theta \omega_{0}\right)\right) \geq d\left(\varphi\left(n_{j}, \widehat{\omega}_{j}, x_{j}\right), A(\Omega)\right)$,

Which contradicts (6). Thus, the asserted convergence (5) must hold .

\section{Comparison of non-autonomous attractors}

The mapping $\phi: \mathbb{Z}^{+} \times \mathbb{X} \rightarrow \mathbb{X}$ defined by $\phi(n, \omega, x):=\left(\theta_{n}(\omega), \varphi(n, \omega, x)\right)$, for each $j=1,2$ and $(\omega, x) \in$ $\mathbb{X}:=\Omega \times \mathbb{R}^{d}$ forms an autonomous semi dynamical system on the extended state space $\mathbb{X}$ with the metric

$d_{\mathbb{X}}\left(\left(\omega_{1}, x_{1}\right),\left(\omega_{2}, x_{2}\right)\right)=d_{\Omega}\left(\omega_{1}, \omega_{2}\right)+d\left(x_{1}, x_{2}\right)$.

Theorem 3.1 Suppose that $\mathcal{A}$ is a uniform attractor of an $\operatorname{RDS}(\theta, \varphi)$ and that $\mathrm{U}_{\omega \in \Omega} A(\theta \omega)$ is precompact in $\mathbb{R}^{d}$. Then the union $\mathbb{A}:=\mathrm{U}_{\omega \in \Omega}\{\omega\} \times A(\theta \omega)$ is the global attractor of the autonomous semi dynamical system $\phi$.

Proof: The $\phi$-invariant of $\mathbb{A}$ follows from the $\varphi$-invariant of $\mathcal{A}$, and the $\theta$-invariant of $\Omega$ via

$\phi(n, \mathbb{A})=\cup_{\omega \in \Omega}\left\{\theta_{n}(\omega)\right\} \times, \varphi(n, \omega, A(\theta \omega))=\cup_{\omega \in \Omega}\left\{\theta_{n}(\omega)\right\} \times A\left(\theta_{n}(\omega)\right)=\cup_{\omega \in \Omega}\{\mu\} \times A(\mu)=\mathbb{A}$.

Since $\mathcal{A}$ is also a pullback attractor and $\cup_{\omega \in \Omega} A(\theta \omega)$ is precompact in $\mathbb{R}^{d}$ (and $\Omega$ is compact too ), the set valued mapping $\omega \rightarrow A(\omega)$ is upper semi-continuous. which means that, $\omega \mapsto F(\omega):=\{\omega\} \times A(\omega)$ is also upper semi-continuous. Hence, $F(\Omega)=\mathbb{A}$ is a compact random subset of $\mathbb{X}$ implies that

$$
\begin{aligned}
& d_{\mathbb{X}}(\phi(n,(\omega, x)), \mathbb{A}) \\
& =d_{\mathbb{X}}\left(\left(\theta_{n}(\omega), \varphi(n, \omega, x)\right), \mathbb{A}\right) \\
& \leq d_{\mathbb{X}}\left(\left(\theta_{n}(\omega), \varphi(n, \omega, x)\right),\left\{\theta_{n}(\omega)\right\} \times A\left(\theta_{n}(\omega)\right)\right) \\
& =d_{\Omega}\left(\left(\theta_{n}(\omega), \theta_{n}(\omega)\right)+d\left(\varphi(n, \omega, x), A\left(\theta_{n} \omega\right)\right)\right.
\end{aligned}
$$


$=d\left(\varphi(n, \omega, x), A\left(\theta_{n} \omega\right)\right)$.

Where $\phi(n,(\omega, x))=\left(\theta_{n}(\omega), \varphi(n, \omega, x)\right)$. The desired attraction to $\mathbb{A}$ with respect to $\phi$ then follows from the forward attraction of $\mathcal{A}$ with respect to $\varphi$.

Theorem 3.2 If $\mathcal{A}$ is a pullback attractor for a random dynamical system $(\theta, \varphi)$ and $\cup_{\omega \in \Omega} A(\theta \omega)$ is precompact in $\mathbb{R}^{d}$, then $\mathbb{A}:=\mathrm{U}_{\omega \in \Omega}\{\omega\} \times A(\theta \omega)$ is the maximal invariant compact random set of the autonomous semi dynamical system $\phi$.

Proof: The $\varphi$-invarianc and compactness of $\mathbb{A}$ are proved in the same manner as in first part of the proof of Theorem 3.1. To prove that the invariant compact set $\mathbb{A}$ is maximal, let $C$ be any other invariant compact set of the autonomous semi dynamical system $\varphi$. Then $\mathbb{A}$ is a $\varphi$-invariant and compact collection of compact sets, and pullback attraction,

$d\left(\varphi\left(\left(n, \theta_{-n}(\omega), \mathcal{C}\left(\theta_{-n}(\omega)\right), A(\theta \omega)\right)\right.\right.$

$\leq d\left(\varphi\left(\left(n, \theta_{-n}(\omega), K(\omega), A(\theta \omega)\right) \rightarrow 0\right.\right.$

as $n \rightarrow \infty$, where $K(\omega):=\overline{\mathrm{U}_{\omega \in \Omega} \mathcal{C}(\theta \omega)}$ is compact. Hence, $\mathcal{C}(\theta \omega) \subseteq A(\theta \omega)$ for each $\omega \in \Omega$, that is, $\mathrm{C}:=$ $\cup_{\omega \in \Omega}\{\omega\} \times \mathcal{C}(\theta \omega) \subseteq \mathbb{A}$, which finally means that $\mathbb{A}$ a maximal $\phi$-invariant set.

Theorem 3.3 If an autonomous semi dynamical system $\varphi$ has a global attractor $\mathbb{A}=\cup_{\omega \in \Omega}\{\omega\} \times A(\theta \omega)$, then $\mathcal{A}=\{A(\theta \omega): \omega \in \Omega\}$ is a pullback attractor for the $\operatorname{RDS}(\theta, \varphi)$.

Proof: The sets $\Omega$ and $K(\omega):=\cup_{\omega \in \Omega} A(\theta \omega)$ are compact by the compactness of $\mathbb{A}$. Moreover, $\mathbb{A} \subset \Omega \times$ $K(\omega)$, which is a compact set. Now

$$
\begin{aligned}
d(\varphi(n, \omega, x), K(\omega)) & =d_{\Omega}\left(\theta_{n}(\omega), \Omega\right)+d(\varphi(n, \omega, x), K(\omega)) \\
& \left.=d_{\mathbb{X}}\left(\theta_{n}(\omega), \varphi(n, \omega, x)\right), \Omega \times K(\omega)\right) \\
& \leq d_{\mathbb{X}}(\phi(n,(\omega, x), \Omega \times K(\omega)) \\
& \leq d_{\mathbb{X}}(\phi(n, \Omega \times D), \mathbb{A}) \rightarrow 0 \text { as } n \rightarrow \infty
\end{aligned}
$$

for each $(\omega, x) \in \Omega \times D$ and every arbitrary bounded subset $D$ of $\mathbb{R}^{d}$, since $\mathbb{A}$ is the global attractor of $\phi$. Hence, replacing $\omega$ by $\theta_{-n}(\omega)$ implies $\lim _{n \rightarrow \infty} d\left(\varphi\left(n, \theta_{-n}(\omega), D(\omega), K(\omega)\right)=0\right.$. Thus , $\mathcal{A}$ is pullback attractor of the $\operatorname{RDS}(\theta, \varphi)$. 


\section{References}

[1] Cheban, D.N., Global attractors of infinite-dimensional nonautonomous dynamical systems, I, Bulletin Acad. Sci. Rep. Moldova. Mathematics, 25 (3) (1997), 42-55.

[2] Cheban, D.N., Nonautonomous dissipative dynamical systems, Differentsial'nye Uravneniya, 23 (3) (1987), 464-474.

[3] Cheban, D.N., Nonautonomous dynamical systems with a convergence, Differentsial'nye Uravneniya, 25 (9): (1989) 1633-1635.

[4] Chepyzhov, V., and Vishik, M.I., Attractors of non-autonomous dynamical systems and their dimension, J. Math. Pures Appl., 73 (1994), 279-333.

[5] Crauel, H., and Flandoli, F., Attractors for random dynamical systems, Prob. Theor. Related Fields, 100 (1994), 365-393.

[6] G. Ochs. Weak random attractors. Technical report, Institut f ur Dynamische Systeme, Universit”at Bremen, 1999.

[7] Haraux. A., Syst’emes Dynamiques Dissipatifs et Applications, Masson, Paris, 1991.(20)

[8] Kloeden, P.E., and Kozyakin, V.S., The inflation of nonautonomous systems and their pullback attractors, Transactions of the Russian Academy of Natural Sciences, Series MMMIU. 4, No. 1-2, (2000), 144-169.

[9] L. Arnold "Random Dynamical Systems", Springer-Verlag Berlin Heidelberg Germany (1998).

[10] M. Scheutzow. Comparison of various concepts of a random atractor: A case study. Technical report, Technische University at Berlin, 2000.

[11] Sell, G.R., Lectures on Topological Dynamics and Differential Equations, Van-NostrandReinhold, Princeton, 1971. 